\title{
La situation politico-linguistique des langues régionales de France: le cas du breton ${ }^{1}$
}

\section{(La situación político-lingüística de las lenguas regionales de Francia: el caso del bretón)}

\author{
Karina Costa Morales² \\ Universidad Nacional, Costa Rica \\ Julio Sánchez Murillo ${ }^{3}$ \\ Universidad Nacional, Costa Rica
}

\begin{abstract}
résumé
Du point de vue linguistique, la France est un pays très divers malgré la suprématie du français. Pour propager cette diversité, des tentatives cherchent à revaloriser les langues régionales, même au niveau constitutionnel. Un cas représentatif de cette tendance est le breton, langue celtique parlée à l'ouest de la France. Dans la région de Bretagne, il existe plusieurs mouvements politico-culturels très actifs dont l'un des objectifs consiste à officialiser la langue bretonne, la répandre et changer son statut et son image auprès des Français et des Bretons. Depuis des lustres, on peut repérer les résultats de leurs actions, à savoir, la place du breton dans le système éducatif et dans les médias et le breton dans le discours politique et le monde culturel. Ces faits marquent une certaine orientation ; cependant, les langues régionales, en particulier le breton, ont des difficultés pour obtenir une stabilité, et leur futur devient douteux.
\end{abstract}

1 Elaborado con base en la ponencia presentada por sus autores en el IV Congreso Internacional de Lingüística Aplicada, llevado a cabo en mayo de 2013, en el Campus Omar Dengo, de la Universidad Nacional de Costa Rica. Recibido: 19 de abril de 2012; aceptado: 3 de julio de 2012.

2 Escuela de Literatura y Ciencias del Lenguaje. Correo electrónico: karine.vanesse@london.com

3 Escuela de Literatura y Ciencias del Lenguaje. Correo electrónico: jesm80@ costarricense.cr

$$
L_{\text {etras }} 52 \text { (2012), ISSN 1409-424X }
$$




\section{resumen}

Desde el punto de vista lingüístico, Francia es un país muy diverso a pesar de la supremacía del francés. Para ampliar esta diversidad, algunos esfuerzos tienden a revalorizar las lenguas regionales, incluso a nivel constitucional. Un caso representativo de esta tendencia es el bretón, lengua celta hablada en el oeste de Francia. En la región de Bretaña, existen varios movimientos político-culturales muy activos cuyo objetivo es oficializar la lengua bretona, extenderla y modificar su estatuto y su imagen en la sociedad francesa y bretona. Desde hace algunos lustros, se pueden palpar los resultados de sus acciones; por ejemplo: el bretón en el sistema educativo y en los medios de comunicación y el bretón en el discurso político y el mundo de la cultura. Estos hechos marcan una cierta orientación; sin embargo, las lenguas regionales, particularmente el bretón, tienen dificultades para obtener una estabilidad y su futuro es incierto.

Mots-clés : langues régionales de France, langue bretonne, politiques linguistiques, enseignement des langues régionales

Palabras clave: lenguas regionales de Francia, lengua bretona, políticas lingüísticas, enseñanza de las lenguas regionales

\section{Introduction}

À l'intérieur de la France, il y a quelques langues qui possèdent des statuts souvent peu connus. Malgré les tentatives mises en pratique de généraliser l'utilisation du français et de déconseiller l'emploi de ces langues régionales pendant le passé, les nouvelles tendances linguistiques recommandent leur récupération, particulièrement liée à leurs représentations culturelles.

La première étape qu'il fallait suivre afin de constituer un processus de récupération de ce qui avait été considéré comme des «patois» ou des versions non standards de la langue qui, pendant cette période, était la seule base de la culture française, consistait à vérifier la situation de ces langues, souvent, oubliées par la société.

De cette manière, tantôt le niveau constitutionnel, tantôt les manifestations cachées démontraient qu'il existait une certaine 
rupture entre ce qui se passait au niveau politique et ce que les gens exigeaient par rapport à la conservation du patrimoine linguistique.

En 1999, le linguiste Bernard Cerquiglini a élaboré un rapport (Les langues de France) pour le Ministère de l'Éducation nationale et le Ministère de la Culture (gouvernement du socialiste Lionel Jospin) où il dénombre plus de 70 langues régionales et minoritaires. Certaines langues font partie actuellement du panorama linguistique français comme conséquence de divers mouvements et vagues migratoires du xx ${ }^{\mathrm{e}}$ siècle (l'arabe dialectal ou l'arménien, par exemple).

Cette étude a permis de réaffirmer la pluralité linguistique de la France et de démontrer de nouveau la situation difficile qu'elles vivent concernant leur statut, leur nombre de locuteurs et leur place dans la société contemporaine.

Dans le tableau suivant ${ }^{4}$, on présente les langues régionales les plus représentatives de la France métropolitaine et de la France d'outre-mer.

4 Les données présentées dans ce tableau, concernant le nombre de locuteurs des langues régionales, ont été prises de la page web du Ministère de la Culture et de la Communication de France. 


\section{Tableau 1. Les langues régionales les plus représentatives de France}

\begin{tabular}{|c|c|c|}
\hline Langues* & Région(s) où elles sont parlées & Locuteurs \\
\hline $\begin{array}{c}\text { Alsacien } \\
\text { (Elsässisch) }\end{array}$ & Alsace & 650000 \\
\hline $\begin{array}{l}\text { Basque } \\
\text { (Euskera) }\end{array}$ & Aquitaine & 50000 \\
\hline $\begin{array}{c}\text { Breton } \\
\text { (Brezhoneg) }\end{array}$ & Bretagne et Pays de la Loire & 206000 \\
\hline $\begin{array}{l}\text { Catalan } \\
\text { (Català) }\end{array}$ & Languedoc-Roussillon & 110000 \\
\hline $\begin{array}{l}\text { Corse } \\
(\text { Corsu })\end{array}$ & Corse & $\begin{array}{l}\text { Entre } 70000 \\
\text { et } 150000\end{array}$ \\
\hline $\begin{array}{c}\text { Créole } \\
\text { (kréol - kréyol - kreyòl) }\end{array}$ & $\begin{array}{l}\text { Martinique, Guadeloupe, Guyane } \\
\text { et La Réunion }\end{array}$ & $2000000^{* *}$ \\
\hline $\begin{array}{c}\text { Flamand } \\
\text { (Vlaemsch) }\end{array}$ & Nord-Pas-de-Calais & $\begin{array}{l}\text { Entre } 30000 \\
\text { et } 50000\end{array}$ \\
\hline $\begin{array}{c}\text { Francique } \\
\text { (Fränkischt) }\end{array}$ & Lorraine & $\begin{array}{l}\text { Entre } 80000 \\
\text { et } 100000\end{array}$ \\
\hline $\begin{array}{c}\text { Franco-provençal } \\
\text { (Arpitan) } \\
\text { Francoprovençâl } \\
\text { (Arpetan) }\end{array}$ & Rhône-Alpes et Franche-Comté & $\begin{array}{l}\text { Entre } 80000 \\
\text { et } 130000\end{array}$ \\
\hline $\begin{array}{c}\text { Occitan } \\
\text { (Occitan ou Lenga d'òc) }\end{array}$ & $\begin{array}{l}\text { PACA, Auvergne, Limousin, Midi- } \\
\text { Pyrénées, Aquitaine, Languedoc- } \\
\text { Roussillon et Rhône-Alpes }\end{array}$ & $\begin{array}{l}\text { Entre } 600000 \\
\text { et } 1600000\end{array}$ \\
\hline $\begin{array}{c}\text { Tahitien } \\
\text { (Reo-tahiti) }\end{array}$ & Polynésie française & 50000 \\
\hline
\end{tabular}

* Entre parenthèses leur nom en langue régionale.

** Ce chiffre correspond aux locuteurs de différents créoles à base lexicale française.

Il est important de mentionner que quelques langues sont parlées dans d'autres pays: le basque, le catalan et l'occitan en Espagne, le franco-provençal en Suisse et en Italie. Le flamand a un grand nombre de locuteurs en Belgique et aux Pays-Bas. Il n'existe pas de langue spécifique dans la Collectivité territoriale de Saint-Pierre-etMiquelon (à l'est du Canada). 
L'analyse de tous les cas de langues régionales en France a permis d'adresser les changements pratiques lors de l'observation d'une nouvelle implémentation de techniques de conservation qui deviendraient peu à peu une sorte de guide pour le gouvernement et les promotions sociales et culturelles.

Cette même analyse s'est focalisée sur une langue renommée en ce qui concerne la préoccupation d'utilisation: le breton. Cette langue nationale affronte aujourd'hui une situation critique qui pourrait être bientôt définie, mais les mesures que l'on attend dépendent de sa situation actuelle que l'on examinera plus bas, par rapport à l'histoire de la langue bretonne, son contexte démolinguistique et son rôle dans les domaines éducatif, médiatique, politique et culturel.

\section{Bref historique de la langue bretonne}

Vers la fin du V $V^{\mathrm{e}}$ siècle de notre ère, un peuple d'origine celte venant de Britannia (aujourd'hui Grande-Bretagne) s'est installé dans la région de l'Armorique (Bretagne actuelle) dans le nord-ouest de la France. Ce mouvement migratoire s'est développé pendant plusieurs siècles.

Ces individus, appelés Bretons, ont apporté avec eux leur culture et leur langue, de cette manière, ils ont contribué à enrichir le paysage linguistique de la région, qui commençait à connaître la domination du latin et la disparition du gaulois.

Cette langue, le breton, fait partie du groupe de langues celtiques, tout comme le gaélique écossais, l'irlandais, le gallois, le manxois, le cornique et d'autres langues qui sont déjà mortes comme le celtibère ou hispano-celtique, parlé dans le territoire actuel de l'Espagne. Ces trésors linguistiques constituent une des principales traces de ce qui a été l'univers celte, un territoire très vaste qui s'étalait sur deux tiers du continent européen, de la péninsule ibérique à la Roumaine, les îles Britanniques et même, la région centrale de la Turquie contemporaine. Ce monde s'est réduit, sous la pression et 
la suprématie d'autres peuples(les Romains et le peuple germanique par exemple), à quelques contrées en Grande-Bretagne, en France et en Irlande. Ces régions sont devenues des îlots linguistiques où les langues celtiques conservent une certaine vivacité.

En ce qui concerne la langue bretonne, elle s'est développée dans les zones rurales de la Bretagne où le latin était faiblement présent et le gaulois s'éteignait inexorablement. Ces faits et le nombre important de Bretons, qui ont immigré dans cette région, ont favorisé l'implantation de leur langue; comme preuve de ceci des documents écrits, tels qu'un texte de botanique: le manuscrit de Leyde ${ }^{5}$, datant du viiie siècle, le plus ancien en breton et conservé à l'Université de Leyde, aux Pays-Bas. Dans ce feuillet de quatre pages, cette langue apparait sous forme des mots techniques (des noms de plantes et de préparations à des fins médicaux), il s'agit surtout de la traduction des noms latins. Ce texte est antérieur aux Serments de Strasbourg.

$\mathrm{Au} \mathrm{x}^{\mathrm{e}}$ siècle, le breton était parlé par la plupart des habitants des régions occidentales, centrales (aujourd'hui bilingues) et des alentours des grandes villes. Néanmoins, cette période d'essor a souffert un recul avec les invasions normandes; malgré ceci, il a été la langue de la classe dirigeante de la région jusqu'au xii siècle, époque à laquelle il a été remplacé par le français.

Un tournant dans l'histoire du breton est la publication du premier dictionnaire français-breton-latin: le Catholicon de Jean Lagadeuc, rédigé en 1464 et imprimé en 1499. Dans ce livre, les mots en breton apparaissaient en ordre alphabétique, puis leur traduction en français et en latin. Voici des exemples tirés d'une édition ${ }^{6} \mathrm{du}_{\mathrm{xix}}{ }^{\mathrm{e}} \mathrm{de}$ ce dictionnaire:

- $\quad$ Eunan, g. seul, l. solus

- $\quad$ Goat, g. sang, l. sanguis

- $\quad$ Lesnau, g. surnom, l. cognomen

5 D'après la page web de l'Office public de la langue bretonne.

6 Édition http://books.google.co.cr/books. 
En 1659, le père Julien Maunoir a publié une grammaire et un dictionnaire français-breton, où il a employé une nouvelle orthographe, celle-ci sera à la base de l'orthographe moderne de la langue bretonne. Dans cet ouvrage, il a introduit le trigramme c'h (prononcé $/ \mathrm{x} /$ ou $/ \mathrm{y} /$, son proche du $\mathrm{j}$ espagnol) pour le distinguer du ch (prononcé /H/) comme en français; par exemple: ar chik (le menton) et ar c'hik (la viande).

Le recul définitif du breton vers l'ouest a commencé après la Révolution Française, dans ce contexte, on ne peut pas passer à côté du discours de Bertrand Berère en 1794: «chez un peuple libre, la langue doit être une et la même pour tous ${ }^{7}{ }^{7}$ En outre, on pourrait citer l'évolution et l'expansion de l'école comme un des phénomènes les plus importants dans l'affermissement du français comme langue de France, au détriment des autres idiomes; le breton a été interdit à l'école et ceux qui osaient l'employer étaient sévèrement punis.

Pendant les $\mathrm{xix}^{\mathrm{e}}$ et $\mathrm{xx}^{\mathrm{e}}$ siècles, des grammaires et des dictionnaires ont vu la lumière et des efforts pour créer un breton standard et unifier cette langue, fragmentée en six variétés; par exemple: le Dictionnaire celto-breton (1821) de Jean-François Le Gonidec. En 1908, un groupe de grammairiens et linguistes bretons a proposé de nouvelles modifications orthographiques et l'unification de trois dialectes de Cornouaille, Léon et Trégor. Dans les années 1940, des écrivains ont proposé une orthographe dite unifiée du breton, en incluant, cette fois, l'autre dialecte: le vannetais. La plupart des publications et des enseignants emploient cette orthographe.

Comme une réaction contre cette proposition, le linguiste et phonéticien François Falc'hun a créé, dans les années 1950, l'orthographe universitaire. Celle-ci a été principalement utilisée par le clergé et les communistes de Cornouaille et Léon.

7 Henriette Walter, Aventures et mésaventures des langues de France (Paris: Champion Classiques, 2012) 67. 
En dépit des efforts postérieurs (1975 et 2003) pour établir une orthographe, les divergences continuent et il existe, alors, plusieurs orthographes du breton en usage.

Aujourd'hui, cette langue constitue le principal symbole de la culture bretonne et malgré le processus d'érosion; il y a quelques décennies, une période d'engouement envers elle a surgi, ce qui a permis de promouvoir son enseignement, sa présence dans les médias et d'assurer en quelque sorte son avenir. Ainsi, les événements concernant le breton et son histoire peuvent être comparés dans ce tableau.

\section{Tableau 2. Résumé des données historiques}

\begin{tabular}{|l|l|}
\hline \multicolumn{1}{|c|}{ Époque } & \multicolumn{1}{c|}{ Événements } \\
\hline Vers le $\mathrm{v}^{\mathrm{e}}$ siècle & Arrivée des Bretons en France. \\
\hline viii $^{\mathrm{e}}$ siècle & Apparition du «manuscrit de Leyden», écrit en breton. \\
\hline $\mathrm{x}^{\mathrm{e}}$ siècle & Le breton est parlé par la plupart des habitants de la région. \\
\hline xii $^{\mathrm{e}}$ siècle & $\begin{array}{l}\text { Déclin du breton en tant que langue dirigeante de la } \\
\text { région, remplacé par le français. }\end{array}$ \\
\hline 1499 & Publication du premier dictionnaire français-breton-latin. \\
\hline 1659 & $\begin{array}{l}\text { Publication d'une grammaire et d'un dictionnaire } \\
\text { français-breton. }\end{array}$ \\
\hline xviii ${ }^{\mathrm{e}}$ siècle & Recul du breton vers l'ouest. \\
\hline xix ${ }^{\mathrm{e}}$ et $\mathrm{xx}^{\mathrm{e}}$ siècles & Efforts pour créer un breton standard. \\
\hline 1940 & Proposition d'une orthographe unifiée du breton. \\
\hline 1950 & Création d'une orthographe universitaire. \\
\hline 1975 et 2003 & Continuation des efforts pour établir une orthographe. \\
\hline
\end{tabular}

\section{Données démolinguistiques}

Le breton est une langue celtique indoeuropéenne parlée dans la région de Bretagne, à l'ouest de la France. Il s'agit, alors, d'une relation linguistique directe tantôt avec le cornique, tantôt avec le gallois, deux langues parlées au Royaume-Uni, ce qui explique l'historique de la langue et des habitants Bretons. 
Le statut actuel du breton correspond à la langue la plus parlée en Bretagne après le français, à partir duquel il a obtenu une reconnaissance en tant que langue régionale ou minoritaire de France.

Cette langue est surtout parlée dans la partie ouest de la Bretagne, où l'organisation de la défense du breton est mieux articulée que dans le sud, où l'on parle d'une certaine dispersion directement liée à la concentration de la population qui a toujours été vinculée aux côtes de la Bretagne. ${ }^{8}$

Des 4.405.572 habitants de Bretagne, seulement 206.000 correspondent à des locuteurs natifs de la langue, c'est-à-dire, à peu près $5 \%$ de la population et de ce pourcentage, les locuteurs de plus de 60 ans atteignent $61 \%$. Ces données ont de sérieuses répercussions sur la future quantité de brittophones. ${ }^{9}$

D'après certaines statistiques, le nombre de locuteurs bretons a beaucoup diminué pendant le $\mathrm{xx}^{\mathrm{e}}$ siècle: en 1914, 90 \% de la population bretonne de l'ouest parlait breton, un chiffre qui en 1945 constituait 75 $\%$ jusqu'à arriver à l'état critique d'aujourd'hui, ce qui représente son statut en tant que langue en péril de disparition et qui reflète l'évolution de la quantité de locuteurs, montrée dans le tableau suivant.

\section{Tableau 3: Évolution du nombre de locuteurs du breton dans les derniers 35 ans}

\begin{tabular}{|c|c|c|c|}
\hline $\mathbf{1 9 7 6}^{\mathbf{1 0}}$ & $\mathbf{1 9 9 4}^{\mathbf{1 1}}$ & $\mathbf{2 0 0 0}^{\mathbf{1 2}}$ & $\mathbf{2 0 0 7}$ \\
\hline $\begin{array}{c}\text { Environ 1 million (dont } \\
\text { la moitié faisait un usage } \\
\text { quotidien) }\end{array}$ & 500000 & $\begin{array}{c}240000 \text { (dont la } \\
\text { moitié l'utilisait } \\
\text { couramment) }\end{array}$ & 206000 \\
\hline
\end{tabular}

$8 \quad$ F. J. Schrijver, Regionalism after Regionalisation: Spain, France and the United Kingdom (Amsterdam: Amsterdam University Press, 2006).

9 http://www.ofis-bzh.org/fr/langue_bretonne/chiffres_cles/index.php, consulté le 10 février 2013.

10 Marc Blancpain et André Reboullet, Une langue le français (Paris: Hachette, 1976) 34.

11 Henriette Walter, L'aventure des langues en occident (Paris: Éditions Robert Laffon, 1994) 116.

12 Henriette Walter (2012) 68. 
Il existe des éléments qui contribuent au développement, à la stagnation ou au recul d'une langue, le linguiste français Louis-Jean Calvet propose dans son livre La guerre des langues et les politiques linguistiques $^{13}$ une série de facteurs exerçant sur les langues une certaine pression; parmi lesquels, la géographie, l'économie, la religion et la politique. La situation actuelle du breton a pu être influencée par ceux-ci.

$\mathrm{Au}$ sujet de l'aspect géographique, cette langue, étant donné que la Bretagne est une péninsule entourée par l'océan Atlantique, a cherché à se répandre vers l'est (où se trouvent les villes de Rennes et Nantes); cependant, dans la zone oriental de la Bretagne historique, le breton a toujours était très faible. L'importance de grandes agglomérations est évidente, les gens qui s'installent dans ces centres administratifs, politiques et économiques doivent adopter la langue qui y prédomine pour accéder au travail, à l'éducation, au système de santé ou au monde de la culture et le breton est surtout une langue rurale.

Pendant longtemps, le breton a été la langue d'évangélisation employée par l'église catholique, cependant suite à la francisation de la région et à l'affaiblissement général de la religion dans la société, le facteur religieux n'est plus un élément déterminant dans l'avenir de la langue.

Le pouvoir économique et politique pourrait jouer un rôle essentiel sur le destin du breton; il est vrai qu'à l'heure actuelle, pour trouver un bon poste dans une grande entreprise, le breton n'est pas nécessaire et que les hommes et femmes politiques n'ont pas besoin de s'adresser à leurs électeurs en breton; cependant, une partie importante de l'avenir de cette langue dépend des décisions politiques, étroitement attachées au facteur économique.

Depuis la déclaration de danger pour le breton, la préoccupation des Bretons a augmenté pour ainsi récupérer les traits de cette langue complètement liée à leur culture, et malgré les mesures mises en pratique, par exemple, l'apprentissage de la part de quelques

13 Louis-Jean Calvet, La guerre des langues et les politiques linguistiques (Paris, Hachette, 2009, troisième édition) 130-134. 
enfants, il est difficile de déterminer si l'utilisation de la langue bretonne se continuera une fois que leur enfance sera terminée. ${ }^{14}$

Bien que le breton soit en danger, il comporte encore des caractéristiques d'une langue naturelle, telles que les dialectes, qui se différencient surtout en ce qui concerne la prononciation et le vocabulaire. Ainsi, on trouve 6 dialectes bretons: le cornouaillais, le léonard, le trégorrois, le vannetais, le breton de Loire-Atlantique et le breton du Goëlo, vinculés à différentes régions. ${ }^{15}$

\section{Éducation}

Dans le domaine de l'éducation, le breton vit des scénarios contradictoires, nous voulons proposer une révision succincte de la place de cette langue dans le système éducatif de la région.

\section{École primaire}

Par rapport à l'éducation continue, l'enseignement primaire est le plus privilégié en ce qui concerne l'enseignement de la langue bretonne et si l'on le compare avec la diminution qui se passe dans l'enseignement secondaire et supérieur. En fait, il y a trois modalités qui incluent ce type d'éducation: les écoles Diwan, la méthode Div Yezh et la Dihun.

La première modalité, les écoles Diwan, a été établie en 1977 avec l'objectif d'enseigner le breton en immersion, et ces écoles sont connues comme des écoles bilingues qui sont devenues populaires en raison de leur niveau d'exigence. Alors, ces écoles avaient 14709 enfants pour la rentrée 2012, avec plus de $25 \%$ de croissance des filières bilingues entre 2007 et 2012, dans 142 communes pourvues de ce type d'enseignement.

14 Yann Fournis, Les régionalismes en Bretagne: la région et l'État (Bretagne: Peter Lang, 2006).

15 <http://www.langues-de-france.org/broudic.html>, consulté le 8 février 2013. 
De sa part, la méthode Div Yezh est utilisée dans plusieurs écoles en tant qu'approche bilingue, mais ce n'est pas aussi populaire que les écoles Diwan. Finalement, la méthode Dihun correspond à une stratégie utilisée dans plusieurs écoles catholiques de la région bretonne à partir de 1990, dans le but de faire appel à un «réveil» par rapport à l'utilisation du breton.

\section{École secondaire}

On trouve un certain déclin de l'enseignement du breton au niveau secondaire, soit parce que l'on trouve peu de collèges qui proposent des cours de cette langue, soit parce que les offres sont peu nombreuses, soit parce qu'il n'y a aucune présence de la langue. Ceci démontre l'un des dangers de la petite continuation de l'enseignement commencé depuis l'école et qui pourrait changer la situation du breton.

Nonobstant, on trouve quelques institutions d'enseignement secondaire qui offrent des cours de breton en tant que langue étrangère et en tant que choix parmi d'autres langues, telles que l'espagnol ou l'allemand. Mais il faut se rendre compte que la situation n'est pas bien définie pour assurer une bonne expérimentation de l'enseignement de la langue.

\section{Enseignement supérieur}

La situation du breton n'est pas du tout risquée dans le cadre de l'enseignement supérieur. 763 étudiants suivent des études de breton en tant que filière indépendante et il y a près de 5000 étudiants aux universités de Bretagne qui choisissent la langue bretonne en tant qu'option de langue étrangère pour accompagner leurs études.

En Bretagne, l'Université de Rennes offre des cours en langue et en culture bretonnes jusqu'au Master, tandis que l'Université Catholique de l'Ouest Bretagne-Sud et l'Université de Bretagne occidentale donnent le choix d'obtenir une licence en études linguistiques du breton. 


\section{D'autres types d'éducation}

Entre les années 2011 et 2012, près de 3640 adultes ont pris la ferme décision d'apprendre le breton en tant que langue étrangère avec des cours de contact direct. En outre, 1196 adultes se sont inscrits en stages de langue bretonne et plus de 400 adultes ont suivi des cours par correspondance.

À part les études primaires pour les enfants, on a, en Bretagne, des études d'initiation en langue bretonne, surtout dans le département du Finistère, où elles comprennent plusieurs heures par semaine et proposent des activités adaptées aux styles d'apprentissage des enfants inclus.

\section{Enseignants}

Les professeurs monolingues ont l'option de devenir des professeurs bilingues s'ils possèdent un Master en rapport avec l'enseignement bilingue ou les langues régionales. Ils doivent, d'ailleurs, valider leur niveau de breton selon le cadre européen commun de référence ou suivre des formations dont la majorité des frais peuvent être payés par les employeurs et il s'agit d'une formation rapide.

En dépit d'une importante croissance du breton et des autres langues régionales dans le système d'enseignement français, surtout à l'école ${ }^{16}$, leur place y reste encore délicate. Dans la dernière réforme éducative, le Projet d'orientation et de programmation pour la refondation de l'école de la République, adopté en juin 2013 par le Sénat prévoit pour ces langues, après les modifications accordées par quelques sénateurs à la proposition originelle du ministre de l'Éducation nationale Vincent Peillon, des mesures qui procureront la reconnaissance de l'enseignement bilingue (français-langue régionale). En outre, les professeurs pourront s'appuyer sur les langues régionales pour faciliter l'étude du socle commun de connaissances. 
Ce nouveau contexte pourrait constituer un soutien considérable à l'avenir de ces langues; pourtant, il faudra attendre quelques années pour voir les résultats de cette réforme et mieux évaluer son rôle dans le développement et stabilisation du breton et des autres langues de France.

\section{La langue bretonne dans les médias}

La présence du breton dans les médias est faible et ceci malgré le dynamisme culturel de la région et les efforts de diverses institutions publiques et associations pour doter cette langue des moyens de diffusion.

En effet, il existe en Bretagne un réseau de systèmes de communication qui permet l'accès à des sources d'information, de connaissance et de divertissement en langue bretonne. Il est certain que ces médias favorisent la revalorisation de cette langue et contribuent à l'essor ou au moins à la stabilisation du breton; il est fort reconnu l'importance des médias, surtout électroniques, dans la survivance d'une langue.

Depuis quelques décennies, la radio joue un rôle primordial dans la diffusion du breton; dans les années 1940, Radio Rennes Bretagne accordait une place relativement importante à cette langue, plus tard, d'autres radios ont commencé à offrir des émissions dans cette langue celtique.

Parmi les stations de radios retransmettant des émissions en langue bretonne, nous pouvons citer: Radio Arvorig FM du département du Finistère dont l'objectif est de promouvoir la langue et la musique celtique. Dans cette radio les émissions parlées sont exclusivement en breton. Dans le département du Morbihan, Radio Bro Gwened diffuse une partie de sa programmation en breton, tout comme Plum'FM, Radio Kerne qui propose 60 heures hebdomadaires en breton, Radio Kreiz Breizh qui offre 7 émissions en langue bretonne et France Bleu Breizh Izel ${ }^{17}$.

17 Renseignements pris des pages web de ces chaînes de radio. 
Dans le monde de la télévision ouverte ou câblée, la position du breton reste fragile, même s'il y est présent depuis les années 1960, « c'est en 1964 qu'est diffusé le premier journal télévisé régional. À l'occasion de ce journal, Charles Le Gall vient, toutes les semaines, faire une chronique en breton d'une minute et demie./ ${ }^{18}$ Actuellement, la langue bretonne dispose de plus de 90 secondes d'exposition télévisée mais sa présence se limite à quelques émissions retransmises par les chaînes France 3 Bretagne, Tébéo (dans le Finistère), Ty Télé (dans le Morbihan), TV Rennes 35 et TV Breizh; la plupart de ces émissions sont circonscrites à divers domaines: l'information, la culture et le divertissement; par exemple: Brezhoneg Bemdez (la langue bretonne), Dibikouz (dessins animés pour les enfants), Foeterien (jeu télévisé), Bali Breizh (actualité bretonne), An Taol Lagad (journal télévisé), entre autres.

Concernant la presse écrite, le breton est moyennement placé. $\mathrm{Au} \mathrm{xix}^{\mathrm{e}}$, il y avait déjà des périodiques en breton ou des éditions bilingues et tout au long $d u x^{\mathrm{e}}$, cette présence s'est accrue. À l'heure actuelle, il existe diverses revues présentant la réalité régionale, nationale ou internationale ou des contenus spécialisés. À titre d'exemples, nous mentionnons Bremañ où l'on aborde l'actualité de la région, $Y a$ (hebdomadaire en langue bretonne) et les revues littéraires et culturelles Al Liamm, Brud Nevez, Al Lanv, Aber, Hor Yezh. En outre, avec une certaine régularité, des articles en breton sont publiés dans les journaux locaux, comme Le Télégramme qui propose une rubrique en breton.

À notre époque, l'Internet joue un rôle essentiel dans la communication, c'est pourquoi il est venu revitaliser la diffusion de cette langue; aujourd'hui, des chaînes de télévision: Brezoweb (exclusivement en breton), ABP TV ou An Tour Tan, des radios et les versions numériques des revues transmettent leur programmation ou publient leurs articles à travers ce moyen. En plus, l'Internet facilite l'accès 
à de nombreuses sources de documents écrits, sonores et visuels, parmi eux, des cours gratuits de langue bretonne.

Les médias occupent une place précieuse dans les stratégies qui tiennent à affermir le breton et assurer son avenir, c'est pour cela qu'il faudrait augmenter et mieux canaliser les effectifs financiers investis par la société et les institutions publiques bretonnes et françaises dans la diffusion de cette langue car sa présence dans le monde médiatique reste limitée et incertaine.

\section{Mouvements politico-culturels de reconnaissance du breton}

L'avenir d'une langue menacée, comme le breton, est difficile d'assurer sans qu'il y ait autour d'elle un mouvement social, culturel et politique qui soutienne et amalgame les désirs, besoins et revendications d'une tranche importante de la population de Bretagne, fière de sa culture et de ses origines mais inquiète du destin de sa langue.

Les défenseurs et promoteurs du breton mènent, de même que les locuteurs du basque, catalan, corse, alsacien et des autres langues régionales, une lutte pour la reconnaissance de leur idiome.

Dans cette voie, il faut dire que les langues régionales sont reconnues dans la constitution de la République Française, l'article 75-1 indique depuis 2008 qu'elles «appartiennent au patrimoine de la France», ceci grâce au soutien de la plupart des députés de gauche comme de droite (communistes, socialistes, écologistes, conservateurs, libéraux). Toutefois, pour les groupes qui se battent pour ces langues, cette déclaration a surtout une valeur symbolique et ils demandent des mesures plus consistantes, des politiques qui permettent d'assurer leur diffusion et enseignement et par la suite, leur avenir.

En 1999, le gouvernement de Lionel Jospin a signé la Charte européenne des langues régionales et minoritaires; cependant, elle n'a pas encore été ratifiée, car le Conseil constitutionnel s'oppose en raison de l'article 2 de la Constitution qui établit que «le français est la langue de la République». D'après le linguiste Claude Hagège, cette situation peut 
s'expliquer par «la tradition centralisatrice [qui] est une très vieille donnée du pouvoir et des mentalités de ce pays. L'idée que la France s'est construite - ce qui n'est pas sans être vrai - autour de la langue du roi, devenue la langue de la République, est déterminante.» ${ }^{19}$

Outre le centralisme français, les contradictions de la classe politique contribuent aussi à renforcer la situation précaire, dans quelques cas, des langues régionales. Pour constater ceci, nous citons les propos de quelques candidats à l'élection présidentielle de 2012: Nicolas Sarkozy (UMP, droite) a affirmé : "Quand on aime la France, on ne propose pas de ratifier la Charte des langues régionales et minoritaires»; Jean-Luc Mélenchon (FG, gauche) veut stimuler l>apprentissage des langues régionales à l’école publique mais souligne que «certains articles de la Charte nuisent à l'unité et à l'indivisibilité de la République ». Pour le Front National (extrême droite), le français doit rester la seule langue de la République. La Charte des langues régionales «n'est qu'une étape vers la reconnaissance des langues de l'immigration. Le mondialisme a besoin de fragmenter les peuples pour qu'ils se noient dans des conflits locaux et intercommunautaires..$^{20} \gg$ Lors de cette élection, François Hollande (PS, centre-gauche) et François Bayrou (MODEM, centre) étaient pour la ratification de la Charte.

En ce qui concerne les partis politiques de sensibilité bretonne, nous trouvons des positions variées, relatives à la langue et à la culture bretonnes et au statut de la Bretagne. Nous voulons présenter les idées rapportées à ces sujets de certains partis représentatifs de la vie politique de la région.

L'Union démocratique bretonne ou Unvaniezh Demokratel Breizh, située à gauche dans l'échiquier politique, prône l'accès effectif des Bretons à leur histoire, leur culture et leur langue; en plus, ce parti défend la réunification de la Bretagne historique et demande

19 Claude Hagège propos recueillis par Olivier Le Naire, «Ne méprisons pas les langues régionales», L'Express, édition numérique, 2007, consulté le 13 février 2013.

20 Propos pris du site http://quoi.info/actualite-societe/2012/03/30/, consulté le 20 février 2013. 
plus d'autonomie pour la région; l'UDB est le plus important des partis régionalistes et son discours est plutôt modéré concernant la question bretonne.

Le Parti Breton ou Strollad Breizh, est un parti centriste qui défend le droit des Bretons de se gouverner eux-mêmes. Ses membres conçoivent la Bretagne comme une nation qui doit avoir un statut particulier permettant aux Bretons de gérer les compétences les plus importantes de la région (éducation, impôts, langues, entre autres) et proposent, en plus, deux langues officielles: le breton et le français.

Dans le paysage politique, il existe d'autres groupes comme le Mouvement Bretagne et progrès (Breizh War Raok) qui soutiennent aussi la diffusion et l'enseignement du breton dans les écoles publiques mais on trouve également des partis radicaux, tels que Breizhistance Indépendance et Socialisme (gauche) qui demande l'émancipation de la Bretagne et l'établissement du breton comme langue de scolarisation et Adsav (Renaissance), organisation politique d'extrême-droite, qui promeut aussi l'indépendance de la région et veut accorder au breton le statut de langue d'État. ${ }^{21}$

On peut constater un intérêt commun pour la défense et diffusion du breton et la préservation de la culture bretonne, en dépit des différences idéologiques. Cependant, ces coïncidences n'ont pas encore réussi à accorder à cette langue un statut plus défini dans le système éducatif ou dans l'administration publique, il est évident qu'il reste à réaliser un travail d'articulation politique régionale permettant de faire évoluer positivement la situation du breton. D'ailleurs, il faudrait que ces organisations assument des positions conjointes au moment de demander aux autorités nationales françaises une politique plus claire et réaliste envers les langues régionales.

Également, il existe en Bretagne un réseau de groupes et institutions culturels qui travaillent pour la langue et la culture bretonnes. Forts de leur sens de responsabilité, au sujet de la préservation de cet

$21 \quad$ D'après les pages web de ces partis politiques. 
héritage, ceux-ci agissent, depuis quelques décennies, pour que ce monde breton ne disparaisse pas, malgré le manque de moyens financiers. Parmi ces organisations, nous pouvons citer le Mouvement breton (Emsav), l'Institut culturel de Bretagne, le Conseil culturel de Bretagne (Kuzul ar Breizhoneg), Emgleo Breizh, Div yezh Breizh, l'Office de la Langue Bretonne et beaucoup d'autres.

De sa part, l'Office de la Langue Bretonne est vinculée aux regroupements voulant l'implémentation du breton dans différents champs, surtout en ce qui concerne le patrimoine de la langue mentionnée et de son étude et développement. Une autre fonction de cet office consiste à adresser des campagnes promouvant le breton chez les entreprises et à l'intérieur des communes, ce qui représente un appui considérable et qui comprend des aspects économiques qui peuvent améliorer la situation linguistique et culturelle du breton. Un exemple de ce qui est réalisé avec la participation des communes est détaillé dans le tableau 4, où on présente des certifications données aux communes selon les actions entreprises pour protéger le breton. Les certifications sont attribuées selon la quantité d'actions, concentrées sur les axes d'affichage, diffusion et utilisation.

\section{Tableau 4: les quatre niveaux de certification ${ }^{22}$}

\begin{tabular}{|l|l|}
\hline Certification «Ya d'ar brezhoneg»01 & réalisation d'au moins 5 actions \\
\hline Certification «Ya d'ar brezhoneg»02 & réalisation d'au moins 10 actions \\
\hline Certification «Ya d'ar brezhoneg»03 & réalisation d'au moins 15 actions \\
\hline Certification «Ya d'ar brezhoneg»04 & réalisation d'au moins 30 actions \\
\hline
\end{tabular}

Il est important d'aborder le rôle de la Délégation générale à la langue française et aux langues de France, cette institution, attachée au Ministère de la Culture et de la Communication, joue aussi un rôle important dans la préservation du breton et des autres langues régionales

22 Selon un document officiel de l'Office de la Langue Bretonne, disponible sur $<$ http://www.ofisbzh.org/upload/ouvrage/fichier/301fichier.pdf>. 
et minoritaires et dans la diffusion des manifestations du type culturel (littérature, théâtre, chanson, productions audiovisuelles, entre autres) liées à ces idiomes.Comme preuve de la vitalité de ces groupes et de l'importance du breton au sein de la société bretonne, le 31 mars 2012, «près de 10000 à 12000 personnes ont défilé dans les rues de Quimper pour la défense des langues régionales.» ${ }^{23}$

Nous constatons qu'un nombre important de Bretons est conscient du besoin de mener à bien une série d'actions sociales et politiques, d'abord pour sauver la langue bretonne, puis pour la stabiliser et finalement pour qu'elle prenne les dimensions d'une langue de communication.

\section{Conclusions}

Il est clair que la situation de nombreuses langues régionales en France, même si elle a beaucoup changé pendant les dernières décennies, constitue une combinaison d'aspects positifs et négatifs qui peuvent déterminer les mesures à prendre dans les années à venir, surtout dans le cas que nous avons examiné, celui du breton.

D'abord, les aspects historiques et démolinguistiques mettent en évidence la perte graduelle de la présence du breton en tant que langue de communication quotidienne dans la région de Bretagne, un problème que les sociétés de défense de cette langue essaient de combattre car il devient le début de la planification.

Ensuite, le domaine de l'éducation, en tant qu'outil d'expansion de la langue, apporte aujourd'hui une certaine croissance pour les niveaux les plus bas; cependant, ceci ne suffit pas parce qu'il faut obtenir des garanties à propos de l'usage quotidien du breton. Il faudra aussi trouver une solution permanente au problème du manque d'enseignants de cette langue.

23 Thomas Brégardis, «Langues régionales. De 10000 à 12000 manifestants à Quimper», OuestFrance: édition numérique, 2012, consulté le 12 février 2013. 
En outre, les médias se sont imprégnés, d'une manière graduelle, de cette préoccupation pour collaborer avec l'utilisation de la langue bretonne. De cette manière, il s'agit d'une technique vraiment importante afin de créer des liens entre la vie quotidienne et l'inquiétude du futur d'une langue qui avait fait partie des échanges de tous les jours au passé.

Concernant le domaine de la culture, il sera nécessaire de prendre des mesures pour développer et promouvoir la littérature et la chanson en langue bretonne. La traduction devrait avoir une place primordiale pour offrir à la société bretonne l'accès à un vaste réseau culturel et scientifique en breton.

Il est évident qu'il manque une législation qui protège cette langue en tant que moyen de transmission de valeurs culturelles et lui accorde une place bien déterminée dans la vie sociale, éducative et administrative de la région.

D'ailleurs, les mouvements qui deviennent de plus en plus forts par rapport aux domaines politiques et culturels représentent, dans une certaine mesure, les désirs des habitants de la région bretonne, et l'on dirait qu'il existe une sorte d'identification du peuple avec l'envie d'une continuation d'un élément étroitement lié aux racines de la région, et cet aspect pourrait être l'un des plus importants.

Les langues régionales de France vivent une situation plus fragile que dans d'autres pays européens, tels que 1'Espagne; alors, on peut déduire que l'avenir de celles-ci passe par une vraie politique linguistique émanant des institutions nationales: le gouvernement, l'Assemblée nationale et le Sénat.

Finalement, bien qu'il y ait des projections qui se concentrent sur le futur du breton, à partir des données que l'on a examinées, il est difficile de déterminer si le futur de cette langue est assuré, mais la continuation avec les mesures que l'on possède aujourd'hui pourrait devenir la clé de sa conservation. 